\title{
Recommendations for the Reporting of Specimens Containing Oral Cavity and Oropharynx Neoplasms
}

\begin{abstract}
Association of Directors of Anatomic and Surgical Pathology
The Association of Directors of Anatomic and Surgical Pathology (ADASP) has named several committees to develop recommendations concerning the content of the surgical pathology report for common malignant tumors. A committee of individuals with special interest and expertise writes the recommendations, which are reviewed and approved by the council of ADASP and subsequently by the entire membership.

The recommendations have been divided into the following four major areas: an informative gross description; additional diagnostic features that should be included in every report, if possible; optional features that may be included in the final report; and a checklist (Table 1). The purpose of these recommendations is to provide an informative report to the clinician. The recommendations are intended as suggestions, and adherence to them is completely voluntary. In special clinical circumstances, the recommendations might not be applicable. The recommendations are intended as an educational resource rather than a mandate.
\end{abstract}

I. Gross description-the Association recommends that the following features be included in the final report because they are generally accepted as being of prognostic importance, required for staging or therapy, and/or traditionally expected.

A. How the specimen was received-fresh, in formalin, oriented by surgeon, etc.

B. How the specimen was identified-labeled (with name, medical record number) and anatomic site designation, e.g., right partial glossectomy, modified neck dissection

C. Describe - portions of oral cavity or oropharynx included with specimen, including other structures that may be attached, e.g., cortical bone of jaws, palate, tongue, skin of neck, maxillary sinus

D. Measure - the overall dimensions of all specimens received

E. Tumor description-size (give in three dimensions), shape (ulcerating, exophytic, polypoid), color, necrosis, multifocal growth

F. Location of the tumor-anatomic sites and subsites

1. External upper lip (vermilion border)

2. External lower lip (vermilion border)

3. Commissures

4. Buccal mucosa

a. Mucosa of upper and lower lips

b. Cheek mucosa

c. Retromolar areas

d. Bucco-alveolar sulci, upper and lower (vestibule of mouth)

5. Upper alveolus and gingiva (upper gum)

6. Lower alveolus and gingiva (lower gum)

7. Hard palate

8. Tongue

Copyright $\odot 2000$ by The United States and Canadian Academy of

Pathology, Inc.

VOL. 13, NO. 9, P. 1038, 2000 Printed in the U.S.A.

Date of acceptance: April 6, 2000.

The members of the ad hoc committee on oral cavity and oropharynx neoplasms are Richard J. Zarbo (Chair), Leon Barnes, M.D., John D. Crissman, M.D.,

Douglas R. Gnepp, M.D., and Stacey E. Mills, M.D. 


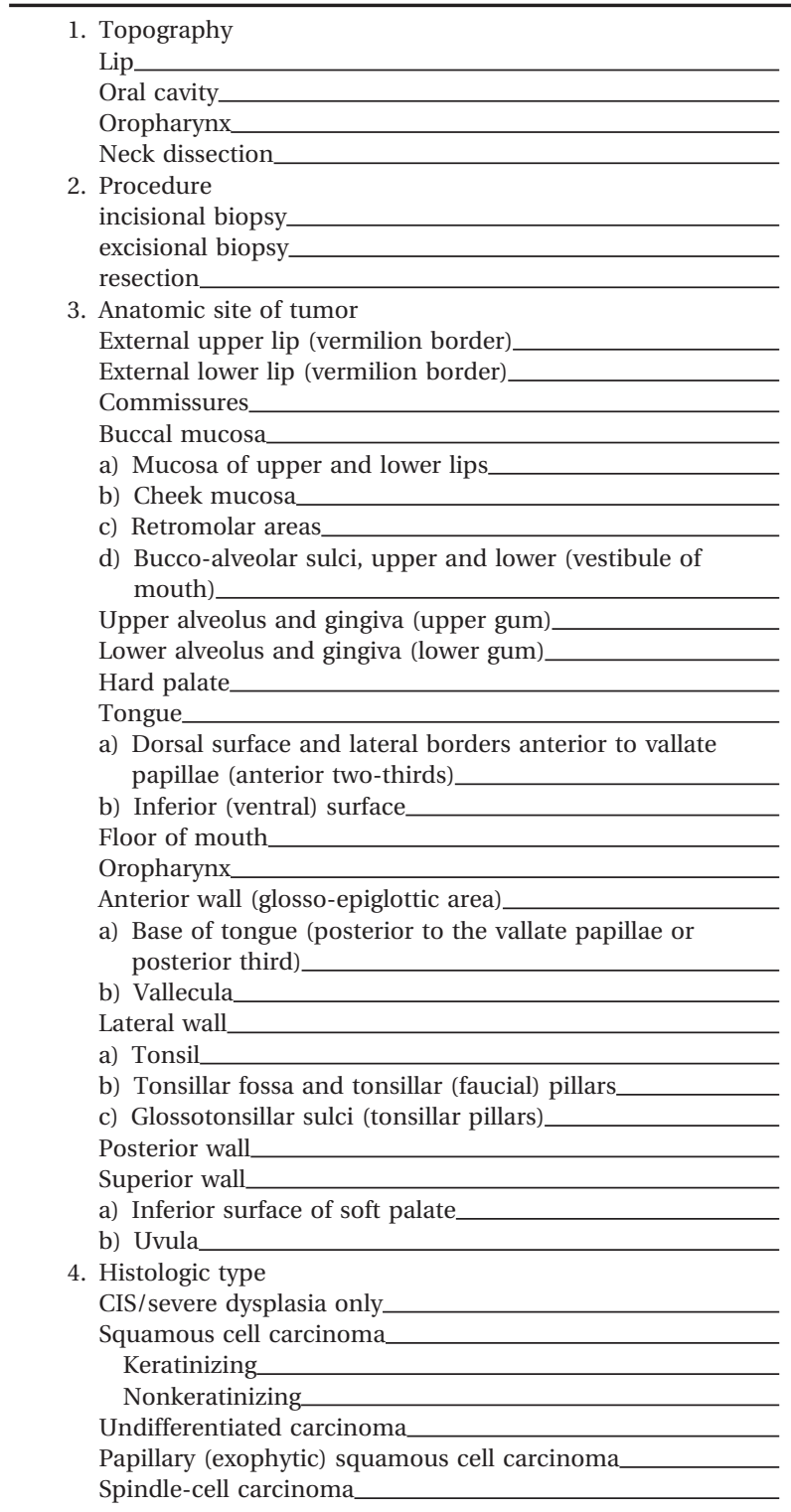

4. continued. .

Verrucous carcinoma

Basaloid squamous-cell carcinoma

Neuroendocrine carcinoma

Well differentiated (carcinoid)

Moderately differentiated (atypical carcinoid)

Poorly differentiated (small cell carcinoma)

Salivary gland carcinoma (specify type)

Adenosquamous carcinoma

Adenocarcinoma, nonsalivary type

Other malignancy (specify)

5. Histologic grade

Well-differentiated

Moderately differentiated

Poorly differentiated

Undifferentiated

6. Tumor extent (see text definitions)

TIS: Carcinoma in situ

T1: Tumor $2 \mathrm{~cm}$ or less in greatest dimension

T2: Tumor more than $2 \mathrm{~cm}$ but not more than $4 \mathrm{~cm}$ in greatest

dimension

T3: Tumor more than $4 \mathrm{~cm}$ in greatest dimension

T4: Tumor invades adjacent structures, e.g., through cortical bone, mandible, inferior alveolar nerve, skin or soft tissues of neck, deep (extrinsic) muscle of tongue, ptergoid muscles, maxillary sinus, hard palate, larynx

Multicentric tumor

7. Status of surgical margins (specify specimen margins or margins separately submitted)

Free of tumor

Involved by tumor (specify)

8. Lymph node metastases (specify right or left)

Number of nodes removed

Number of nodes involved

Size of largest involved node

Extracapsular invasion present

Jugular vein invasion present

Muscle invasion present

Keratin debris and/or foreign body giant cell reaction present

9. Preoperative treatment effects on nodes

Yes

No

10. Special investigations performed

Flow cytometry

Electron microscopy

Image analysis

Molecular diagnostics

Gross photograph

a. Dorsal surface and lateral borders anterior to vallate papillae (anterior two-thirds)

b. Inferior (ventral) surface

9. Floor of mouth

10. Oropharynx

a. Anterior wall (glosso-epiglottic area)

i. Base of tongue (posterior to the vallate papillae or posterior third)

ii. Vallecula

b. Lateral wall

i. Tonsil

ii. Tonsillar fossa and tonsillar (faucial) pillars

iii. Glossotonsillar sulci (tonsillar pillars)

c. Posterior wall

d. Superior wall

i. Inferior surface of soft palate

ii. Uvula 
G. Tumor extent-based on tumor classification (AJCC, UICC) (applicable only to carcinomas of the vermilion surfaces of the lips and of the oral cavity and oropharynx, including those of minor salivary glands) $(1,2)$

1. All sites

a. TIS-carcinoma in situ

b. T1-tumor $2 \mathrm{~cm}$ or less in greatest dimension

c. T2-tumor more than $2 \mathrm{~cm}$ but not more than $4 \mathrm{~cm}$ in greatest dimension

d. T3-tumor more than $4 \mathrm{~cm}$ in greatest dimension

e. $\mathrm{T} 4$

- Lip-tumor invades adjacent structures, e.g., through cortical bone, inferior alveolar nerve, floor of mouth, skin of face.

- Oral cavity - tumor invades adjacent structures, e.g., through cortical bone, into deep (extrinsic) muscle of tongue, maxillary sinus, skin (superficial erosion alone of bone/tooth socket by gingival primary is not sufficient to classify a tumor as T4).

- Oropharynx-tumor invades adjacent structures, e.g., ptergoid muscles, mandible, hard palate, deep muscle of tongue, larynx

2. Note 1-The extrinsic musculature of the tongue includes musculi hypo-, stylo-, genio- and palatoglossus. Invasion of the intrinsic muscle alone (musculi longitudinales superior and inferior, transversus linguae and verticalis linguae) is not classified T4

3. Note 2-in cases of doubt regarding the invasion through cortical bone, Paragraph 4 of the General Rules of the TNM System (TNM Booklet, p. 6) should be applied: "If there is doubt concerning the correct $\mathrm{T}, \mathrm{N}$ or $\mathrm{M}$ category to which a particular case should be allotted, the lower (i.e., less advanced) category should be chosen. This will also be reflected in the stage grouping." If scintigraphy is feasible and the resultant finding is conclusive the tumor must be classified as T4 (3)

H. Lymph node dissection if included-type (extended radical, radical or modified radical or selective); inclusion of sternomastoid muscle/submandibular and/or parotid gland/jugular vein; palpable mass (solitary, matted); size and location of gross invasion of adjacent soft tissues, muscle, and jugular vein; measure and describe sternomastoid muscle, major salivary glands, and internal jugular vein; measure size of lymph nodal masses (see Note 3); label lymph nodes as to levels or according to anatomic location in neck dissection

1. Note $3 \mathrm{a}-\mathrm{it}$ is generally recognized that most masses greater than $3 \mathrm{~cm}$ in diameter are not single lymph nodes but represent confluent nodes or tumor in soft tissues of the neck

2. Note $3 b-$ histologic examination of a selective neck dissection specimen will ordinarily include 6 or more lymph nodes. Histologic examination of a radical or modified radical neck dissection specimen will ordinarily include 10 or more lymph nodes (depending on previous RT)

\section{Diagnostic information}

A. Topography—type of specimen(s) received, e.g., simple excision, composite resection, neck contents

B. Procedure- - e.g., total or partial glossectomy, radical neck dissection

C. Exact site of tumor-lip, oral cavity, oropharynx (see Table 1, anatomic site of tumor)

D. Histologic type-World Health Organization classification recommended (see Note 4) (comment on no tumor present post therapy)

1. Note 4-histologic type (World Health Organization Classification, modified) (3) includes squamous cell carcinoma, typical, keratinizing or nonkeratinizing, invasive or in situ; spindle-cell squamous (sarcomatoid) carcinoma; verrucous carcinoma; basaloid squamous cell carcinoma; papillary squamous cell carcinoma; undifferentiated carcinoma (including lymphoepithelioma); salivary gland-type tumor (adenoid cystic carcinoma, mucoepidermoid carcinoma, adenosquamous carcinoma, and others); neuroendocrine carcinoma [well-differentiated (carcinoid tumor), moderately differentiated (atypical carcinoid tumor), poorly differentiated (small cell carcinoma)]; adenocarcinoma, nonsalivary gland type; other malignancies (sarcoma, melanoma, etc.) 


\section{E. Histologic grade as appropriate}

F. Tumor extent-size and depth of invasion with respect to adjacent structures (e.g., tonsillar pillar, soft palate, nasal cavity, ptergoid muscles) extrinsic muscle of tongue, skin and soft tissue of neck and face. Distinguish extending to or overlying bone from gross erosion of bone and radiographic destruction of bone. Note tracheostomy involvement, as well as multifocal growth

G. Status of surgical margins

H. Lymph node metastases - size of metastatic node, number of involved nodes, level of node involvement, comment whether extranodal spread of tumor is found, comment on keratin debris and/or foreign body giant cell reaction as evidence of previous tumor.

I. Preoperative treatment-effects on nodes

III. Optional pathologic features that can be included if desired
A. Extent and location-of any dysplasia (including grade)
B. Vascular/lymphatic invasion
C. Perineural invasion
D. Depth of invasion
E. Interface with stroma-infiltrating, pushing, superficial or deep invasion
F. Inflammatory infiltrate - type of density
G. Results of ancillary investigations-i.e., flow cytometry
H. Distance-from surgical margins

\section{BIBLIOGRAPHY}

1. Sobin, LH, Wittekind, C, editors. International Union Against Cancer (UICC). TNM classification of malignant tumors. 5th ed. New York; Wiley-Liss; 1997.

2. Fleming ID, Cooper JS, Henson DE, et al., editors. American Joint Committee on Cancer (AJCC). AJCC Cancer staging manual, 5th ed. Philadelphia: Lippincott Raven; 1997.
3. World Health Organization. WHO international histological classification of tumors. Pindborg JJ, et al. Histologic typing of cancer and precursors of the oral mucosa. 2nd ed. Berlin: Springer-Verlag; 1992.

4. McClatchey KD, Zarbo RJ. The jaws and oral cavity. Chapter 18. In: Sternberg, SS editor. Diagnostic surgical pathology. 2nd ed. New York: Raven; 1994. p. 759-806. 